\title{
TRAVELING WAVE ULTRASONIC MOTOR USING THIN-FILM PIEZOELECTRICS
}

\author{
Ryan Q. Rudy ${ }^{1,2^{*}}$, Gabriel L. Smith ${ }^{1}$, Don L. DeVoe ${ }^{2}$, and Ronald G. Polcawich ${ }^{l}$ \\ ${ }^{1}$ U.S. Army Research Laboratory, Adelphi, Maryland, USA \\ ${ }^{2}$ University of Maryland, College Park, Maryland, USA
}

\begin{abstract}
The authors report demonstrated bi-directional rotary motion of a millimeter scale traveling wave ultrasonic motor (TWUM) using solution deposited thin film lead zirconate titanate (PZT) and MEMS fabrication techniques. Rotation speeds of 2300 RPM have been achieved at $10 \mathrm{~V}$ and less than $7 \mathrm{~mW}$ in a $500 \mu \mathrm{m}$ thick, $2 \mathrm{~mm}$ diameter rotor. Design, fabrication, and initial testing of the TWUM are described. The low-power, high-torque, zero-power off state rotary motors described here, could enable numerous applications in the fields of small-scale robotics, fuzing, and biomedical technology.
\end{abstract}

\section{INTRODUCTION}

The traveling wave ultrasonic motor (TWUM), reported by Sashida in 1983 [1] holds may advantages over electromagnetic or electrostatic motors including high-torque low-speed operation, and a high holding torque with zero power input [2]. Because the torque output is high at low speeds, the need for gears is eliminated, thus reducing noise and complexity, and creating a more compact design. These benefits can be clearly observed in macro-scale TWUMs used in auto-focus/manual-focus camera lenses [3]. Without the requirement of gears, these near silent TWUMs can be composed of two parts: a stator and a rotor, both of which can be compactly contained within the lens barrel. For miniaturization of motors, the compactness and simplicity of the TWUM is very attractive, especially if one is using MEMS techniques, which offer micron-scale resolution but require largely two-dimensional designs. Because the TWUM exploits friction for the drive mechanism, a high holding torque can be achieved while consuming zero power in an off state. Furthermore, using thin film lead zirconate titanate (PZT) allows for low voltage operation near $5 \mathrm{~V}$ while the high impedance nature of the piezoelectric actuation results in low power consumption when the motor is active.

Large-scale TWUMs, such as the Canon auto-focus lens, have been commercially developed, however effective miniature TWUMs have not been commercialized despite the many advantages they present [4]. Previous work in the area of mm-scale traditional TWUMs has been reported by Flynn [5, 6]. A key improvement between this work and Flynn's is that the devices reported here successfully demonstrate bi-directional rotary motion, whereas the thin-film devices reported by Flynn are unidirectional [5]. The devices reported here are significantly smaller than the assembled bulk PZT devices reported by Flynn in [6]. Kaajakari et al. more recently reported devices which use a bulk PZT driven die to excite parametric resonances which produce motion [7]. These devices however have lost some of the main benefits of traditional TWUM, specifically, a zero-power hold torque, and gearless operation. Our designs maintain these advantages. We have previously reported on our work in characterizing the stator component of the motor [8] and are reporting rotational motion results for the first time here. In the present work we use high quality PZT thin films for wafer-level batch fabrication of hundreds of motors per wafer while exploiting photolithographic patterning for increased manufacturing precision and lower cost. We have demonstrated a compact motor smaller than $3 \mathrm{~mm}$ in diameter with a height of $550 \mu \mathrm{m}$, most of which is inactive substrate. Bi-directional rotor motion has been characterized as well as the effect of input voltage on power consumption and rotational speed.

\section{DESIGN AND FABRICATION \\ Conceptual Design}

The design of our small scale TWUM utilizes solution deposited PZT thin films approximately $1 \mu \mathrm{m}$ thick. Integrated batch fabrication will be realized through wafer bonding of a PZT on silicon-on-insulator (SOI) stator wafer and a rotor wafer. An exploded view of the conceptual design is shown in Fig. 1. In this design, the columns protruding from the stator act to amplify surface motion to increase speed. The preloaded slip ring provides a downward force which increases the torque output by increasing the frictional load. The slip ring also confines the rotor laterally. The stator is supported from the center node point and the electrical connections are made by vias through the base anchor.

\section{Fabrication Process}

Traditional small-scale TWUMs produced using macro-scale manufacturing techniques are bounded in size due precision and accuracy limitations inherent in the fabrication process. Using photolithographic processing and MEMS fabrication techniques these limitations are circumvented and a millimeter-scale TWUM becomes realizable. To demonstrate the rotary motion, stators were fabricated using the process flow in Fig. 2. The process starts with an SOI wafer with a device layer ranging from 20 to $40 \mu \mathrm{m}$ thick and a buried oxide thickness of $1 \mu \mathrm{m}$, shown in Fig. 2 (a). The actuator stack is deposited next, consisting of a 500nm plasma enhanced chemical vapor deposited silicon dioxide thin film, a sputtered titanium thin film annealed to form $33 \mathrm{~nm} \mathrm{TiO}$, a $100 \mathrm{~nm}$ sputtered platinum film, chemical solution deposited PZT with a $\mathrm{Zr} / \mathrm{Ti}$ ratio of $52 / 48$ and a thickness of $1000 \mathrm{~nm}$, and another $100 \mathrm{~nm}$ layer of sputtered platinum as shown in Fig. 2 (b). The top platinum, PZT, and bottom platinum are then patterned using argon ion-milling. To access the bottom $\mathrm{Pt}$, a hole is patterned by

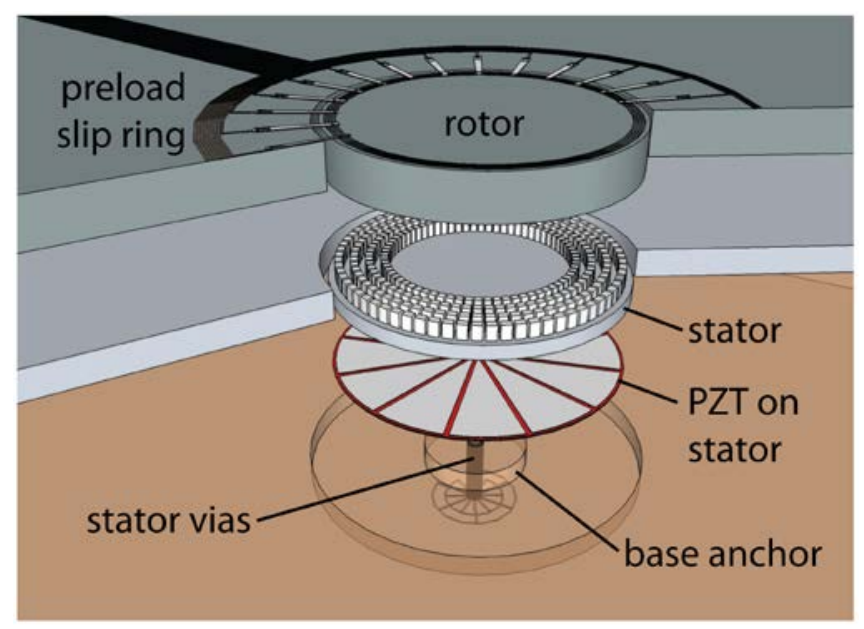

Figure 1: Exploded view of a PiezoMEMS-based TWUM showing the assembled motor. 
a)

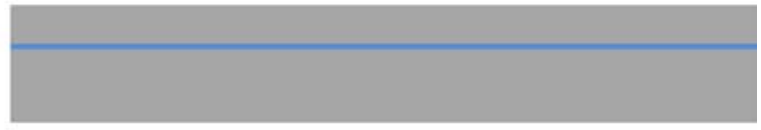

b)

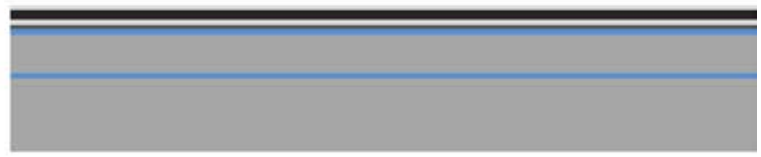

c)

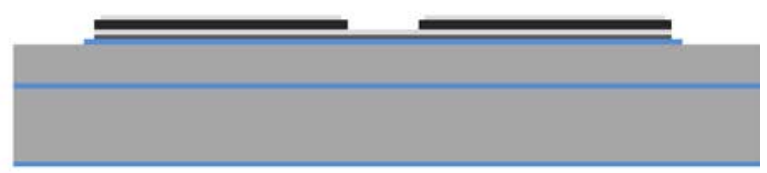

d)

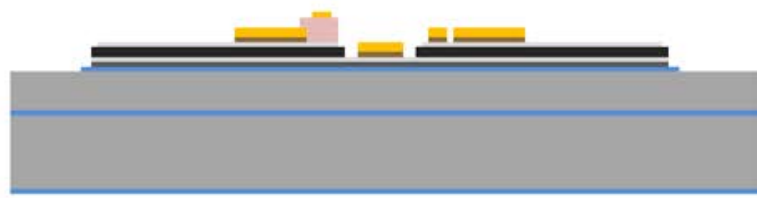

e)

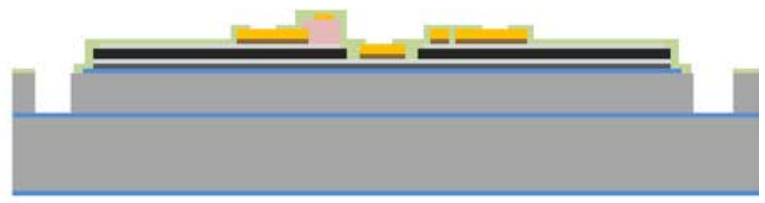

f)

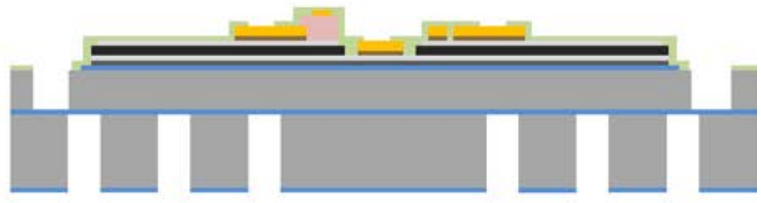

g)

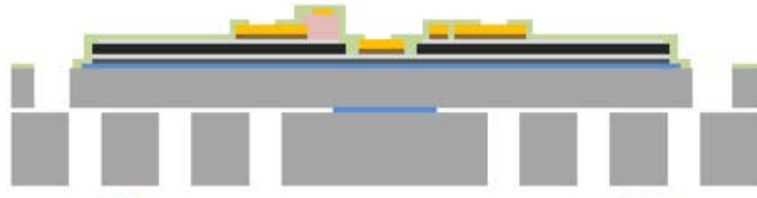

h)

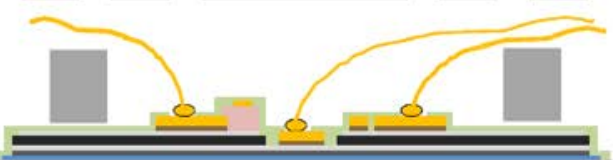

\section{T.}

$\mathrm{Al}_{2} \mathrm{O}_{3}$

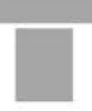

Au

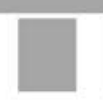

- $\mathrm{Pt}$

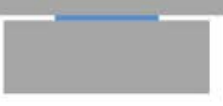

PZT

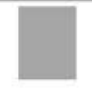

- $\mathrm{Si}$ a lift-off process. A sacrificial layer of photoresist is deposited and patterned to define the gap for gold air bridges. A $2 \mu \mathrm{m}$ thick gold air bridge layer is then deposited via electron beam evaporation and patterned using lift-off, as shown in Fig. 2 (d). The air bridges allow one wirebond to address the three separate drive electrodes of the PZT actuators. A $150 \mathrm{~nm}$ conformal coating of $\mathrm{Al}_{2} \mathrm{O}_{3}$ is deposited using atomic layer deposition to protect the top oxide and PZT during vapor HF release. Following deposition, the $\mathrm{Al}_{2} \mathrm{O}_{3}$ is then etched using Argon ion milling followed by a deep reactive ion etch (DRIE) of the device silicon layer to define the stator device as shown in Fig. 2 (e). Through holes are then etched into the back of the wafer using DRIE to expose the buried oxide as depicted in Fig. 2 (f). The wafer is diced and individual die are released using vapor HF to remove the buried oxide and free the device layer silicon stator as shown in Fig. 2 (g). Once released, the device dies are attached to a package using double-sided Kapton tape. A silicon rotor, fabricated using DRIE, is then placed onto the stator and gold wirebonds from the package are brought through the center of the rotor and attached to the stator electrodes, as shown in Fig. 2 (h). These wirebonds serve two purposes: bringing electrical signal to the stator and constraining the motion of the rotor. A micrograph of the assembled proof of concept system is shown in Fig. 3

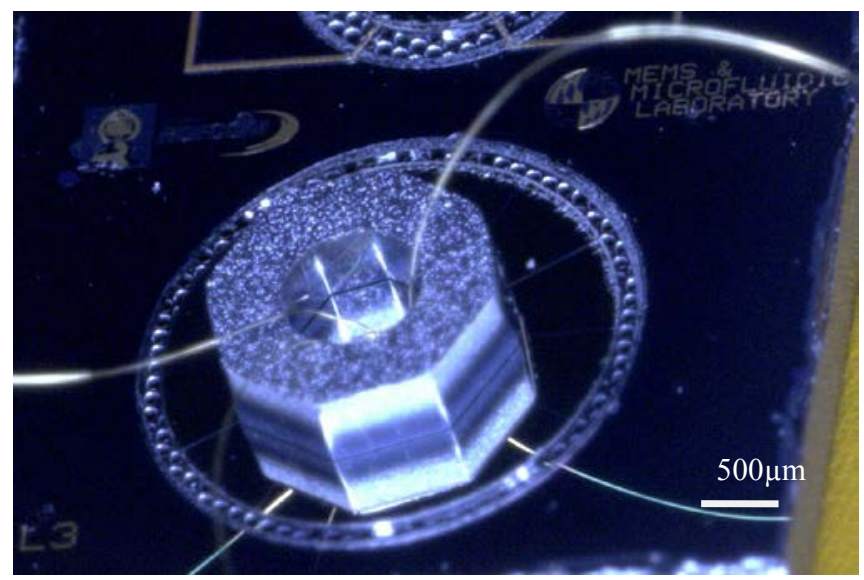

Figure 3: Proof of concept system with a PZT-on-SOI stator with a silicon rotor less than $3 \mathrm{~mm}$ in diameter.

\section{MOTOR OPERATION \\ Operational Theory and Modeling}

TWUMs function by exciting a traveling wave in the stator that propagates around the center-fixed disk at a high frequency. As the wave travels around the disc, the surface points on the disc move in an elliptical trace. This surface motion on the stator then transfers torque through frictional coupling to the free-moving rotor. The traveling wave is created by combining through superposition two identical resonance modes shifted a quarter wavelength in space and offset $90^{\circ}$ in phase. The $\mathrm{B}_{13}$ resonance mode, with one nodal circle and three nodal diameters, was chosen for actuation because the low actuation frequency ensured that we could visualize the wave propagation using a laser Doppler vibrometer (LDV), and the low order mode reduced the number of electrodes that needed electrical connection.

To determine the resonance frequency of the center fixed stator disc, finite element modal analysis was performed. Fig. 4 shows the $\mathrm{B}_{13}$ mode shape simulated using ANSYS, with an associated resonance frequency for a $3 \mathrm{~mm}$ diameter, $30 \mu \mathrm{m}$ thick 


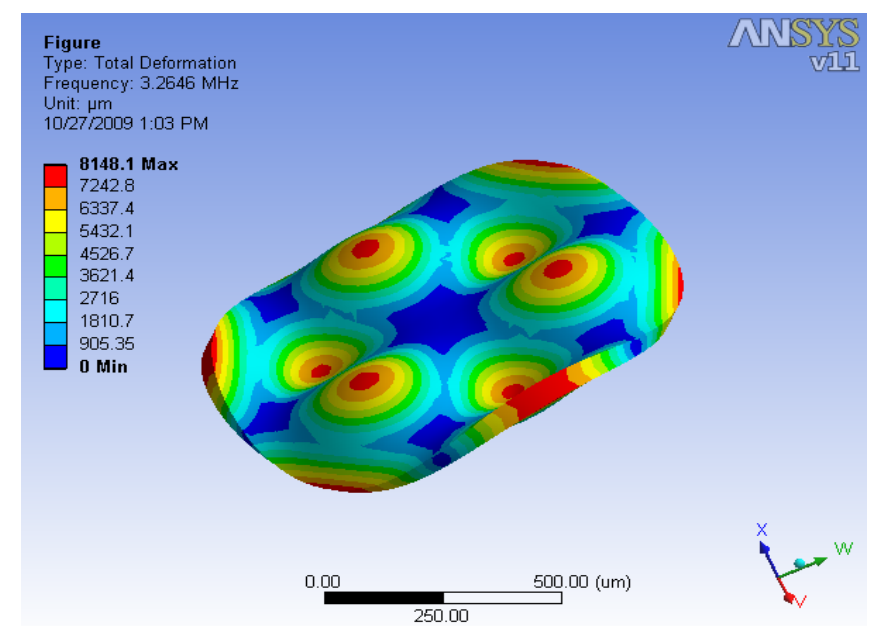

Figure 4: ANSYS modal analysis showing the $B_{13}$ mode.

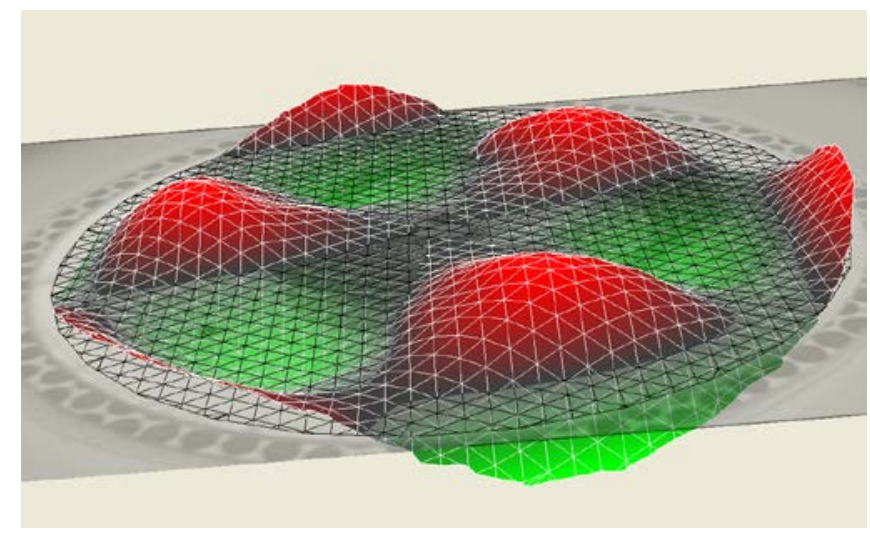

Figure 5: LDV data showing $B_{13}$ mode excited at a frequency of $252 \mathrm{kHz}$.

disc of $284 \mathrm{kHz}$. Experimental results confirmed the model with the $\mathrm{B}_{13}$ resonance mode occurring at $252 \mathrm{kHz}$. The displacement profile of the $B_{13}$ mode, measured using a Polytec LDV, is shown in Fig. 5. According to these measurements, the vibration amplitude of the stator disc is over $1 \mu \mathrm{m}$ at $252 \mathrm{kHz}$. A second set of electrodes excites an identical mode shape with nodal lines at the anti-nodes of the first set. This set-up creates the offset in space to create a traveling wave.

\section{Motor Demonstration and Characterization}

The assembled motor is operated using two actuation electrodes and one ground electrode. The actuation electrodes are offset in phase $90^{\circ}$ and located apart in space to create a propagating wave. Constant rotation begins at $5 \mathrm{~V}$ actuation, where inertial and frictional effects are overcome. Fig. 6 shows the clockwise motion of the rotor in a time-stepped progression. Black dots on the rotor help track the rotation from image to image.

The direction of rotation can be switched by switching the phase offset of the actuation signals from $+90^{\circ}$ to $-90^{\circ}$. Change of direction at $10 \mathrm{~V}$ actuation (1500rpm) has been measured to occur within $30 \mathrm{~ms}$, which highlights the torque output, even without a normal load beyond the weight of the $500 \mu \mathrm{m}$ silicon rotor. Since the motor operates through frictional coupling, increasing the applied normal force increases the torque output of the motor.

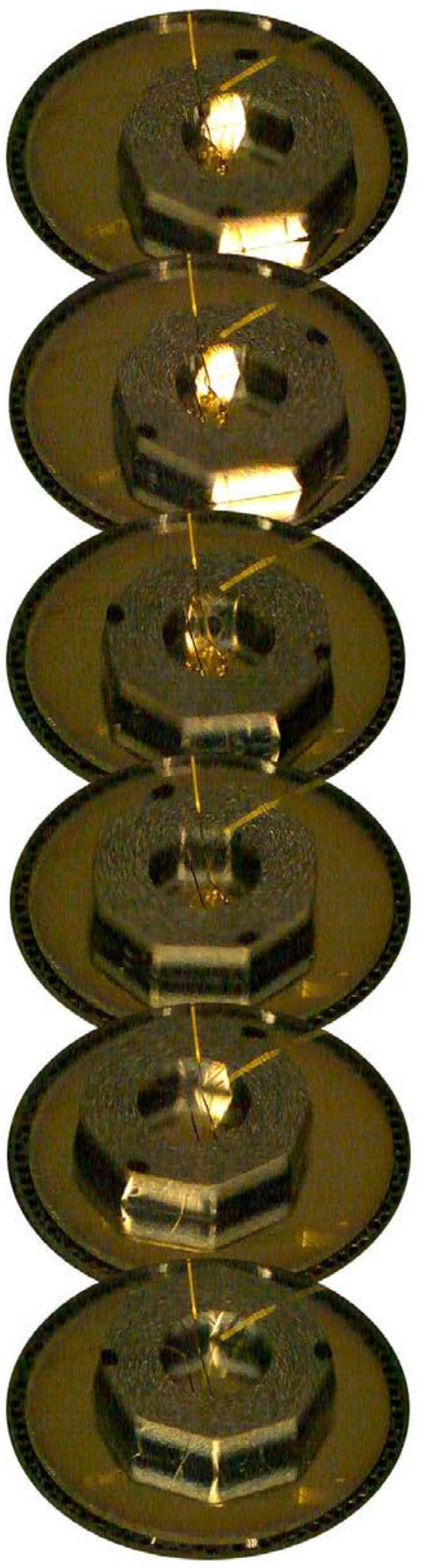

Figure 6: Time-stepped micrographs of $2 \mathrm{~mm}$ rotor spinning at $200 \mathrm{rpm}$. 


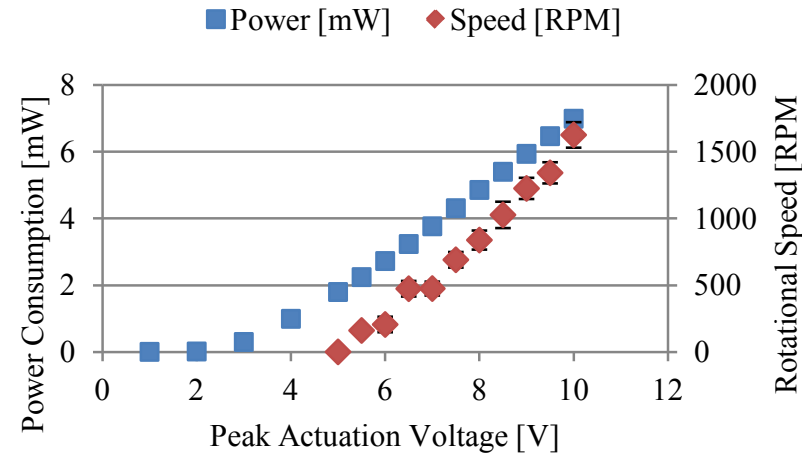

Figure 7: Experimental results showing speed and power as a function of actuation voltage. Error bars on rotational speed represent standard deviation.

At an actuation voltage of $10 \mathrm{~V}$, the wave amplitude is approximately $1 \mu \mathrm{m}$ and current draw from both electrodes totals $0.82 \mathrm{~mA}$ at steady state. Under these conditions, the motor operates near $1500 \mathrm{rpm}$ while drawing $7 \mathrm{~mW}$ of power. Rotational speed and power as a function of actuation voltage are shown in Fig. 7. Maximum rotation speeds, up to $2300 \mathrm{rpm}$, were achieved when the rotor was not in contact with the wirebonds. It should be noted that the breakdown field for the thin-film PZT is significantly higher than applied actuation field, meaning that higher speeds could be achieved with higher applied voltages.

In the assembled motor, the normal force that supports the frictional load is simply the weight of the $500 \mu \mathrm{m}$ thick silicon rotor. Due to the relatively small load applied, estimates of torque are not easily determined, however current designs are being investigated that would allow us to control the normal force and thus tune the output torque.

\section{CONCLUSIONS}

Rotary motion, with bi-directional control, of a $3 \mathrm{~mm}$ diameter TWUM has been demonstrated using thin film PZT and MEMS processing techniques. The characterized proof-of-concept motor shows promise in high rotation speeds, up to 2300rpm and low power, while also providing a substantial lower bound in torque generation and demonstrating the compactness of TWUMs. The stator portion of the motor was modeled and the experimental results compared favorably to this model. This demonstration opens doors to new realms of actuation that have until now been unrealizable, namely high-torques at low-speeds for small-scale actuation. Areas of application include small-scale robotics, low power fuzing, and biomedical devices.

\section{ACKNOWLEDGMENT}

The authors would like to acknowledge Luz Sanchez, Brian Power and Joel Martin of the U.S. Army Research Laboratory for device fabrication. Ryan Rudy would like to acknowledge the SMART fellowship program for support.

\section{REFERENCES}

[1] T. Sashida, "Approach of the Development for the Ultrasonic Motor", Mechanical Automation Japan, vol. 15, no.12, pp.31$35,(1998)$.

[2] T. Maeno, T. Tsukimoto, A. Miyake, "The contact mechanism of an ultrasonic motor," IEEE 7th International Symposium on Applications of Ferroelectrics, (1990), pp. 535-538.

[3] K. Uchino, "Piezoelectric Ultrasonic Motors: Overview", Smart Material Structures, vol. 7, p.273-285 (1998).

[4] K. Uchino, and J.R. Giniewicz, "Micromechatronics", pp.417430 (2003).

[5] A.M. Flynn, et al., "Piezoelectric Micromotors for Microrobots", Journal of Microelectromechanical Systems, vol. 1, no. 1, pp.44-51, (1992).

[6] A.M. Flynn, "Performance of Ultrasonic mini-motors using design of experiments" Smart Material Structures, vol. 7, pp.286-294, (1998).

[7] V. Kaajakari, and A. Lal, "Micromachined Ultrasonic Motor Based on Parametric Polycrystalline Silicon Plate Excitation", Sensors and Actuators A: Physical, vol. 137, no. 1, pp. 120128, (2007).

[8] G.L. Smith, R.Q. Rudy, R.G. Polcawich, and D.L. DeVoe, "Integrated Thin-Film Piezoelectric Traveling Wave Ultrasonic Motors", Sensors and Actuators (2012), 10.1016/j.sna.2011.12.029.

\section{CONTACT}

*R.Q.Rudy, tel: +1-301-394-2324; ryanrudy@umd.edu 